\title{
BMJ Open Protocol for the development of versions of the Montreal Cognitive Assessment (MoCA) for people with hearing or vision impairment
}

\author{
Piers Dawes, ${ }^{\oplus 1}$ Annie Pye ${ }^{2}$ David Reeves, ${ }^{3}$ Wai Kent Yeung, ${ }^{4}$ Saima Sheikh, ${ }^{4}$ \\ Chyrssoula Thodi, ${ }^{5}$ Anna Pavlina Charalambous, ${ }^{5}$ Kathleen Gallant, ${ }^{6}$ \\ Ziad Nasreddine, ${ }^{6}$ Iracema Leroi ${ }^{7}$
}

To cite: Dawes P, Pye A, Reeves D, et al. Protocol for the development of versions of the Montreal Cognitive Assessment (MoCA) for people with hearing or vision impairment. BMJ Open 2019;9:e026246. doi:10.1136/ bmjopen-2018-026246

- Prepublication history for this paper is available online. To view these files, please visit the journal online (http://dx.doi org/10.1136/bmjopen-2018026246).

Received 23 August 2018 Revised 18 January 2019 Accepted 5 February 2019

Check for updates

(c) Author(s) (or their employer(s)) 2019. Re-use permitted under CC BY-NC. No commercial re-use. See rights and permissions. Published by BMJ.

For numbered affiliations see end of article.

Correspondence to

Dr Piers Dawes;

piers.dawes@manchester.ac.uk

\section{ABSTRACT}

Introduction Hearing and vision impairments are highly prevalent among older adults and impact commonly used cognitive assessment tools for the identification of dementia. Adaptations of such tests for people with hearing or vision impairment have not been adequately validated among populations with such sensory impairment.

Methods and analysis We will develop two versions of the Montreal Cognitive Assessment (MoCA) for people with acquired hearing impairment (MoCA-H) or vision impairment (MoCA-V). The MoCA-H and MoCA-V will exclude the existing MoCA items that are presented in spoken or visual format, respectively, and include new suitably adapted items. Participants $(n=792)$ with combinations of hearing, vision and cognitive impairment will complete standard or adapted versions of the MoCA across three language sites (English, French and Greek). Development of the MoCA-H and the MoCA- $\mathrm{V}$ will be based on analysis of adapted and standard MoCA items following model-based development to select the combination of items for the MoCA-H and MoCA-V that provide optimal sensitivity and specificity for detection of dementia.

Ethics and dissemination The study has received ethical approval from respective centres in the UK, France, Greece and Cyprus. The results of the study will be disseminated through peer-reviewed publication, conference presentations, the study website (https://www.sense-cog. eu/), the SENSE-Cog Twitter account (@sense_cog) and the MoCA test website (https://www.mocatest.org/). The main outputs of the study will be versions of the MoCA that are appropriate for use with adults with acquired hearing or vision impairment and will contribute significantly to the clinical care of older people.

\section{INTRODUCTION}

Commonly used tests for cognitive impairment mostly consist of items presented in the visual and/or auditory modality and rely on good sensory function. People with hearing or visual impairment and simulated hearing or vision impairment perform more poorly on tests of cognition than those with normal
Strengths and limitations of this study

- Development and validation of adapted versions of the Montreal Cognitive Assessment (MoCA) for people with acquired hearing or vision impairment will be completed in three languages (Greek, French and English), enabling the cultural validation of both novel and existing versions of the MoCA.

- The MoCA has been shown to have good reliability and validity in screening for cognitive impairment.

- Hearing and vision assessment would be carried out in participants' homes, with background noise and light levels being monitored and controlled during data collection and analysis.

- The validation includes dementia only; validation of the MoCA for people with hearing impairment and the MoCA for people with visual impairment in relation to mild cognitive impairment is planned for the future.

sensory function. ${ }^{1-6}$ The confounding of cognitive tests by hearing or vision impairment may lead to false positive identification of cognitive impairment and/or over-estimation of the severity of cognitive impairment. ${ }^{7}$ Hearing and vision impairment commonly co-occur with cognitive impairment in older adults. In two UK studies, hearing impairment was identified in $94 \%$ of people with a cognitive impairment attending a memory clinic $^{8}$ and a national survey identified visual impairment (visual acuity worse than 6/12) in $32.5 \%$ of a sample of people with dementia. ${ }^{9}$

Previous attempts to adapt cognitive tests for people with sensory impairment involved deleting or substituting written versions of hearing-dependent items and deleting or substituting spoken or tactile versions of vision-dependent items. ${ }^{10}$ Unfortunately, deletion of hearing-dependent or vision-dependent items may adversely impact sensitivity and specificity of the adapted tests. 
To address the need for reliable screening measures of cognitive function for people with acquired sensory impairment, we propose to develop and validate versions of the Montreal Cognitive Assessment (MoCA). ${ }^{11}$ The MoCA is a widely used screening measure that is available free of charge and has been translated into 55 different languages. The MoCA consists of a single page, 30-item test that measures abilities in eight domains: visuospatial/ executive, naming, memory, attention, language, abstraction, delayed recall and orientation. Administration time is usually less than $20 \mathrm{~min}$. The MoCA has previously been validated in populations with vascular dementia, frontotemporal dementia, ${ }^{12}$ Parkinson's disease ${ }^{13}$ and Alzheimer's disease $(\mathrm{AD})^{14}$ and has good sensitivity and specificity for the detection of both dementia and mild cognitive impairment (MCI). ${ }^{11}$

There have been two previous attempts to adapt the MoCA for people with hearing impairment. Lin et al developed a computerised visual version of the MoCA with verbal instructions converted into visual instructions. ${ }^{15}$ Adults with normal hearing $(\mathrm{n}=103)$ or severe-toprofound hearing loss (HL) $(n=49)$ completed the visual version of the MoCA. All participants were screened to have normal cognitive function. Lin et al reported no difference in computerised visual MoCA scores between those with normal hearing and those with hearing impairment. There were no data about the sensitivity and specificity of the computerised visual MoCA for detection of impaired cognitive function.

Dupuis $e t a t^{4}$ also developed a version of the MoCA for people with hearing impairment via deletion of hearing-dependent items from the standard MoCA (language repetition, attention to letters, digit span and delayed recall) to create the MoCA-H. Dupuis $e t a l^{4}$ tested adults with HL (audiometric thresholds $>25 \mathrm{~dB} H \mathrm{HL} ; \mathrm{n}=43$ ) and normal hearing $(n=79)$. The MoCA-H had a higher pass rate than the standard MoCA among people with HL ( $71 \%$ vs 53\%), but fewer people with HL achieved passing scores with proportionally adjusted cut-off scores (to account for the deleted items) versus the normal hearing group (53\% vs $85 \%$ ). The authors concluded that the MoCA-H reduced but did not eliminate poorer performance of hearing impaired versus non-impaired participants.

In relation to adaptations for vision impairment, Wittich et $a l^{3}$ reanalysed data from the original validation of the MoCA to examine the effect of deleting vision-dependent items on sensitivity and specificity for detection of MCI and AD. Wittich et $a \hat{l}$ reported that the MoCA-Blind (involving deletion of four vision-dependent items; trailmaking, copy-cube, clock drawing and picture naming) had increased specificity compared with standard MoCA, but sensitivity was poorer for both MCI and $\mathrm{AD}(63 \%$ and $94 \%$, respectively).

Dupuis et $a l^{4}$ examined performance of the MoCA-Blind on the performance of participants with normal vision $(\mathrm{n}=259)$ versus those with vision impairment (based on far acuity poorer than $<$ logarithm of the minimum angle of resolution ( $\operatorname{LogMaAR})$ 0.3; $\mathrm{n}=38$ ). There was no significant difference in MoCA-Blind scores between those with normal vision and those with vision impairment.

There are several drawbacks with previous adaptations of the MoCA for sensory impaired populations. First, deleting hearing-dependent or vision-dependent items is liable to compromise the validity of the MoCA, because deletion may lead to particular cognitive domains being under-represented or unrepresented. For example, all the hearing-dependent questions that were deleted in the MoCA- $\mathrm{H}^{4}$ relate to memory. It would be preferable to substitute items in an alternative sensory modality rather than deleting items. ${ }^{10}$ Second, no studies have validated adapted versions of the MoCA in terms of sensitivity and specificity to detect cognitive impairment among people with sensory impairment.

This protocol describes development and validation of hearing-independent and vision-independent versions of the MoCA with respect to discrimination between normal cognition and dementia. Hearing-independent and vision-independent versions of the MoCA will be developed based on substitution rather than deletion of items. Furthermore, hearing-independent and vision-independent versions of the MoCA will be validated in English, Greek and French following the translation procedure outlined by Cha et al. ${ }^{16}$ Participants with no sensory impairment will complete the standard version of the MoCA in addition to adapted items from the MoCA designed to accommodate either hearing or vision impairment. Participants with hearing or vision impairment will complete the respective adapted version of the MoCA for hearing or vision impairment.

\section{STUDY AIMS}

The objective is to develop two amended versions of the MoCA (version 8.1) adapted to the needs of people with (1) hearing impairment and (2) vision impairment, termed here the MoCA-H and MoCA-V, respectively. These versions will exclude the existing MoCA items that are presented in spoken or visual format, respectively, and include new suitably adapted items. The nature and number of the substitute items are to be determined by empirical investigation, but the goal is that the structure of the MoCA-H and MoCA-V will closely resemble the standard MoCA in terms of the cognitive domains assessed, number of items, scoring and completion time.

\section{METHODS}

\section{Research design}

All participants will be tested for hearing, vision and cognitive function (figure 1). Participants with age associated acquired hearing or vision impairment will complete adapted versions of MoCA (version 8.1) designed to account for hearing or vision impairment, respectively. Participants with normal sensory function will complete the standard version of the MoCA (version 

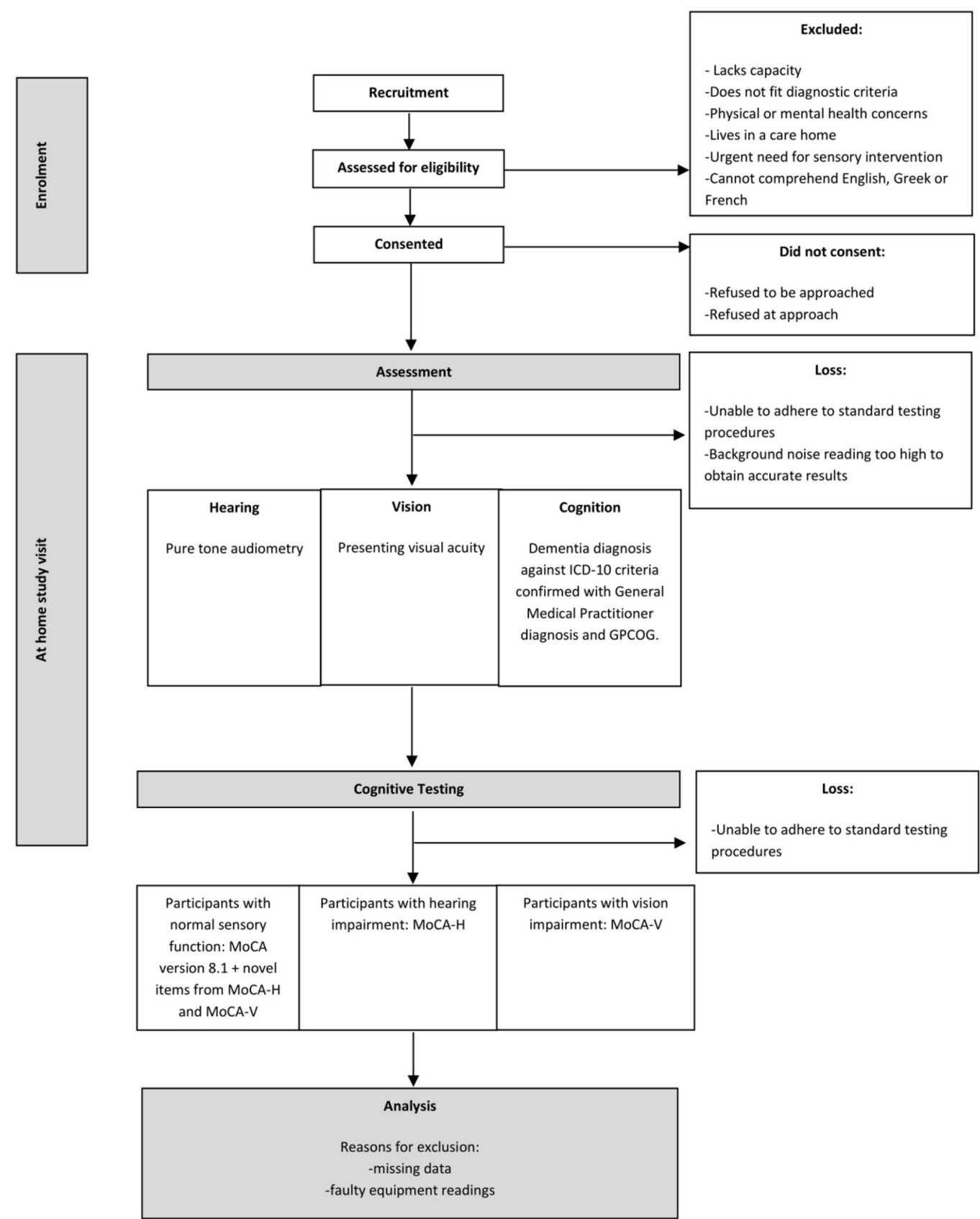

Figure 1 Patient pathway through study. GPCOG, General Practitioner Assessment of Cognition; ICD-10, International Classification of Disease version 10; MoCA, Montreal Cognitive Assessment.

8.1) as well as novel items for the hearing-independent and vision-independent MoCA versions. Adaptations to standard items will be designed to assess the same cognitive domain of the hearing-dependent or vision-dependent items. Adaptation of hearing-dependent items will be done by presenting written instructions and/ or visual versions of items requiring spoken presentation of stimuli. Adaptation of vision-dependent items involves using spoken or tactile versions of items that are presented visually. Two novel scales will be compiled for individuals with hearing and vision impairment, respectively, using the combination of items with the optimal discriminative power to differentiate between normal cognitive function and cognitive impairment. An ideal solution would be one in which each hearing/vision sensitive item in the MoCA is replaced by a single alternative item without affecting the MoCA's domain makeup, reliability or thresholds for determining cognitive impairment. A slightly less preferred solution is one where a single substitute is identified for each hearing/ vision sensitive item, maintaining the domain structure and reliability, but thresholds for identifying cognitive impairment are different. A third and least preferred solution involves a mix of adapted items that do not replicate the existing domain structure (eg, some domains are measured with more or less items than previously) with thresholds for determining cognitive impairment that are different to the standard MoCA. The planned 
Table 1 Overview of participant numbers in each of the study groups

\begin{tabular}{|c|c|c|c|c|c|c|c|}
\hline & $\begin{array}{l}\text { No cognitive } \\
\text { or sensory } \\
\text { impairment }\end{array}$ & $\begin{array}{l}\text { No cognitive } \\
\text { impairment } \\
\text { and hearing } \\
\text { impairment }\end{array}$ & $\begin{array}{l}\text { No cognitive } \\
\text { impairment } \\
\text { and visual } \\
\text { impairment }\end{array}$ & $\begin{array}{l}\text { Dementia and } \\
\text { no sensory } \\
\text { impairment }\end{array}$ & $\begin{array}{l}\text { Dementia } \\
\text { and hearing } \\
\text { impairment }\end{array}$ & $\begin{array}{l}\text { Dementia } \\
\text { and vision } \\
\text { impairment }\end{array}$ & Total \\
\hline Athens & 22 & 22 & 22 & 22 & 22 & 22 & 132 \\
\hline Bordeaux & 22 & 22 & 22 & 22 & 22 & 22 & 132 \\
\hline Bradford & 11 & 11 & 11 & 11 & 11 & 11 & 66 \\
\hline Nicosia & 22 & 22 & 22 & 22 & 22 & 22 & 132 \\
\hline \multirow[t]{2}{*}{ Manchester } & 33 & 33 & 33 & 33 & 33 & 33 & 198 \\
\hline & & & & & & & 792 \\
\hline
\end{tabular}

analysis (see below) is designed to assess, compare and select between these possible solutions.

\section{Participants}

The study will be run in six European sites: Athens, Bordeaux, Nice, Nicosia, Bradford and Manchester. Seven hundred and ninety-two older adults and their significant others/study partner will be recruited into the study in total. The sampling frame (table 1) is designed to provide a balanced sample of participants both with and without dementia and also with and without hearing or vision impairment. One hundred and thirty-two individuals will be recruited into each of six groups (table 1). With the exception of the UK, each site will recruit 22 dyads into each of the six groups and 132 dyads in total. Due to local service limitations, Bradford in the UK will recruit only 11 from each group ( $\mathrm{n}=66$ in total), and Manchester will correspondingly increase recruitment of people across all six groups $(\mathrm{n}=198)$.

\section{Inclusion criteria: primary participant}

All participants will be over 60 years of age and able to provide informed consent to participate in the study. All participants will be living within the community. Participants living in residential care homes and non-domestic settings as individuals who do not comprehend written and spoken English, Greek or French, as well as those with dual sensory impairment (ie, both hearing and vision impairment, according to the definitions of hearing and vision impairment for the study) and those who are culturally deaf or blind will not be included in the study. Participants will also be excluded if they do not have an eligible study partner.

Criteria for the dementia group are based on International Classification of Diseases, 10 th revision criteria ${ }^{17}$ operationalised as: (1) a formal diagnosis of AD, vascular or mixed dementia confirmed via the participant's general medical practitioner and (2) a score within the clinical range (a total score of 0-4) on the General Practitioner Assessment of Cognition $\left(\mathrm{GPCOG}^{18}\right)$. If results on the GPCOG examination are within the borderline range (5-8), the GPCOG informant report will be used to determine the presence or absence of dementia. A score between 0 and 3 on the informant report GPCOG indicates dementia. If a participant scores within the normal range on the GPCOG (9) and/or the GPCOG informant report (4-6), they would be allocated to the 'normal cognition' group. Diagnosis of dementia is restricted to $\mathrm{AD}$, vascular and mixed dementia as these subtypes of dementia account for around $90 \%$ of total dementia diagnoses. ${ }^{19}$ Less common dementia types such as frontotemporal dementia, Parkinson's disease and dementia with Lewy bodies will not be included due to the limited statistical power to conduct analyses of dementia subtypes.

Determination of hearing impairment will be based on pure tone air conduction thresholds in both ears. Individuals with a threshold of greater than $40 \mathrm{~dB}$ HL for the audiometric average of pure tone detection thresholds at $1 \mathrm{kHz}, 2 \mathrm{kHz}$ and $4 \mathrm{kHz}$ will be considered to be hearing impaired. Vision impairment will be based on a measured presenting distance visual acuity of less than 6/12. Any individual who has had fluctuating or recent changes in hearing or visual function will be excluded.

\section{Inclusion criteria: study partner}

The study partner must be over 16 years of age and must have known the primary participant for at least the previous 5 years in order to be able to complete the informant version of the GPCOG.

\section{Sample size calculation}

The sample size is based on achieving acceptably precise estimates of the sensitivity and specificity of the adapted tools for detecting dementia, separately for people with hearing impairment and with vision impairment in relation to the MoCA-H and MoCA-V, respectively. The sample of 264 individuals (132 with dementia and 132 without) within the MoCA-H and MoCA-V groups will enable estimation of the sensitivity to detect dementia and specificity to exclude normal cognition to within $9 \%$ of the true value (based on the exact 95\% CI for a binary variable, calculated using Stata V.15). After combining across all impairment groups, sensitivity and specificity for each of the three language versions will also be estimated to within $9 \%$. 


\section{Recruitment}

Participants will be recruited from ophthalmology and audiology services, memory clinics, volunteer databases and the general community. In the UK, participants will also be recruited through the 'Join Dementia Research' volunteer database. ${ }^{20}$ Sites in France, Greece and Cyprus will develop their own recruitment strategies in accordance with local service provision. The member of the clinical care team at each recruitment site will provide information about the study to potential participants. Potential participants would then contact the research team to arrange participation. Participants will be given a minimum of 24 hours to decide whether they wish to participate in the study.

\section{Consent and testing procedures}

All study visits will take place at participants' homes. At the start of the initial study visit, capacity to consent will be evaluated, and written informed consent will be obtained from both the individual participating and their study partner. All individuals taking consent will have received training in checking capacity in accordance with the legal requirements for conducting research in each country (ie, the Mental Capacity Act [2005] in the UK, the Code de la santé publique in France, Article 47 of the Hospital Law of 1992 (2071) in Greece and article 14 of Law No. 1 (I) 2005 in Cyprus ${ }^{21}$ ). Consent will be considered on an ongoing basis. If more than one study visit is required, willingness to continue will be discussed at the start of each visit with both the older adult and their significant other.

Following informed written consent, participants would complete the GPCOG, and study partners would complete the GPCOG informant version. Participants would then complete hearing and vision assessments before completing the MoCA. Participants in the 'vision impairment' groups would complete the MoCA-V, and participants in the 'hearing impairment' group would complete the MoCA-H. Participants with normal sensory function would complete the standard MoCA as well as the novel items from both the MoCA-V and the MoCA-H. The MoCA-H and the MoCA-V will follow standard MoCA testing procedure as closely as possible.

All data collectors in the study will be trained in Good Clinical Practice ${ }^{22}$ and will have received relevant training on the administration of the screening measures and cognitive tests. Individuals will also have received training on assessing capacity in older adults consistent with relevant local legislation at partner sites. Any individual deemed to be lacking in capacity will not be included in the study. All data transferred between sites will be encrypted, and no individual will be identifiable from the stored data. Identifiable patient information will be stored in a locked cabinet, which will only be accessible to research members at the site of the data collection. Data will be monitored as it comes in for consistency. Data integrity checks will be performed whereby $5 \%$ of all data will be checked against source documents for accuracy.

\section{Patient and public involvement}

Four research user groups (RUGs) of people with dementia with age-related hearing and/or vision impairment and their support people were established in the UK, France, Cyprus and Greece to provide advice on the research. ${ }^{23}$ Research awareness training was provided to support involvement (based on the EQUIP training package ${ }^{24}$ ). RUGs were consulted with respect to: (i) recruitment materials and study documentation and (2) a dissemination plan for the research. Recruitment materials were revised according to RUG feedback to improve readability. The dissemination plan included face-to-face public engagement events and YouTube video summaries following suggestions from the RUGs.

\section{Data statement}

Data will be held in the University of Manchester institutional repository. Published outputs will include a digital object identifier number, and fully anonymised data would be publicly available.

\section{Test-retest}

Five participants from each of the study groups (per language site; $n=30$ ) will be invited to perform a retest of the study measures 2-4weeks after the initial testing. At each site, following a run-in period of 10 participants, consecutive participants will be invited to undertake a retest until the target of five has been achieved.

\section{Assessments}

Hearing and vision

Hearing testing will involve pure tone audiometry using a R07A Screening Portable Audiometer (Kamplex Limited, London), using audiocup headphones (Amplivox, Eden Prairie, Minnesota, USA) to minimise interference from background noise. A KM6 Sound level metre (Kamplex Limited) will be used to measure background noise to ensure that noise levels are below those recommended based on American National Standards Institute standards. ${ }^{25}$ Testing will begin with the self-reported better ear should the participant have one. Participants would be tested without hearing aids, if they use them. Vision testing involves assessment of presenting visual acuity (ie, assessed with glasses that are usually worn for distance viewing) with LED 930 illuminated 3 metre charts (Precision Vision, Woodstock, Illinois, USA). Illuminated charts will be used so that testing can be carried out without additional lighting in order to homogenise light levels within the home environments.

\section{General Practitioner Assessment of Cognition}

The GPCOG is intended as a screening instrument for dementia in primary care settings. The GPCOG and GPCOG informant report versions take less than 4 min to administer. The GPCOG is at least as effective as the MiniMental State Examination ${ }^{26}$ in identifying dementia. ${ }^{27}$ The GPCOG is not impacted by the cultural or linguistic background of the test-taker (although it has not been specifically validated with French or Greek populations), ${ }^{28}$ 
making it an ideal reference for the present cross-national validation study.

\section{Adaptation of MoCA (version 8.1) for people with acquired hearing impairment (MoCA-H)}

Adaptation involved presentation of instructions and stimuli from the MoCA items in written rather than spoken format (table 2). Test-takers will be asked to read the written instructions aloud to the examiner. Research using written versions of cognitive tests has previously demonstrated similar performance to verbal versions. ${ }^{1} 1529$ Two items-'language' and 'attention to letters'-required substitution with alternative items. The 'language' item in the MoCA involves repetition of spoken sentences. Alternative MoCA-H 'language' items involve constructing sentences from a list of visually presented words. The 'attention to letters' item in the MoCA requires test-takers to tap their finger in response to hearing an 'A' in a string of letters that are read aloud. The MoCA-H substitute 'attention to letters' items require participants to read the numbers that are in circles as opposed to squares in a string of numbers bordered by different shapes.

\section{Adaptation of the MoCA (version 8.1) for people with acquired vision impairments (MoCA-V)}

Adaptation of the MoCA for people with vision impairment involved substitution of the first two sections of the MoCA, which rely on good vision (trail making test [TMT], copy cube, clock draw and naming task; table 2). These visually dependent items were substituted for analogous tasks in the auditory domain: visual TMT was substituted with the oral TMT. ${ }^{30}$ The clock draw task was substituted with the Verbal Clock Test. ${ }^{31}$ Both the oral TMT and the Verbal Clock Test are measures of executive function that were designed to remove confounding effects of impaired vision and motor skills on performance and have established validity and reliability. The 'copy cube' task was substituted with questions about the shape of a cube. The 'naming' task was substituted with object identification based on touch. The latter two substitutions were novel items developed by the authors.

\section{Statistical analysis}

The following describes the statistical analysis plan for development of the MoCA-H and the MoCA-V, based on replacing the hearing/vision-sensitive items with adapted items (see table 2). As a first step, each MoCA-H and MoCA-V item will be assessed for the following:

1. Discrimination: no more than $80 \%$ of participants achieving the same score.

2. Feasibility: no more than $5 \%$ missing responses.

3. Redundancy: correlations with other items $>0.75$.

4. Independence from hearing/vision ability: degree of association with level of hearing impairment, based on comparison between item performance between non-sensory impaired groups and hearing-impaired/ vision-impaired groups.
5. Comparability between versions: where relevant, we will compare performance (\% achievement) on the original MoCA item and the adapted version(s) of the item. For the novel items, this will be a within-person comparison based on the data from the non-impaired subgroups collected specifically for this purpose. For other adapted items (eg, where the adaptation involved the provision of written instructions), it will be a comparison between the appropriate non-impaired and sensory-impaired subgroups.

\section{Substitution-based model development}

The substitution of items with written rather than spoken instructions has the potential to change scores. Therefore, the substitution-based analysis will focus on the reliability and score characteristics of the overall instrument, rather than of the individual question items. We will begin by including all adapted items in the instrument scoring and examine the distribution of overall scores, reliability and optimum cognitive impairment threshold scores together with area under the curve (AUC), sensitivity and specificity (via receiver operating characteristic curve analysis). Focusing on the domains where we have multiple alternative adapted items, we will then use a stepwise 'backwards elimination' method to remove items from these domains one by one, in a way that maximises the AUC (as an index of overall predictive performance) without unduly affecting the tool's reliability coefficient. Where there is no clear choice of item for removal, we will also take into account each item's performance indices from the item analysis.

This stepwise procedure will be continued until the adapted instrument has precisely one-to-one substitution of adapted items for original items in every domain throughout, or until it is not possible to remove further items without seriously undermining the level of reliability. The performance measures for the resulting instrument will then be computed.

\section{Exploratory-based model development}

We will also conduct a purely exploratory analysis to identify a version of the MoCA-H and the MoCA-V with the highest degree of discriminative ability between people with and without dementia, regardless of domain makeup. This analysis will follow more standard 'classical' procedures for scale development. From the results of the item analysis, items showing good discrimination, feasibility, low redundancy, comparability and independence from hearing or vision ability will be retained. Items poor on any of these criteria will be considered for removal prior to further analysis. In the case of the MoCA-H, for example, we anticipate that the four existing MoCA hearing-sensitive items will demonstrate association with hearing impairment but will also check and, if necessary, remove additional items.

Following the removal of poorly performing items, we will apply logistic regression to identify the subset of remaining items that best predicts each participant's 


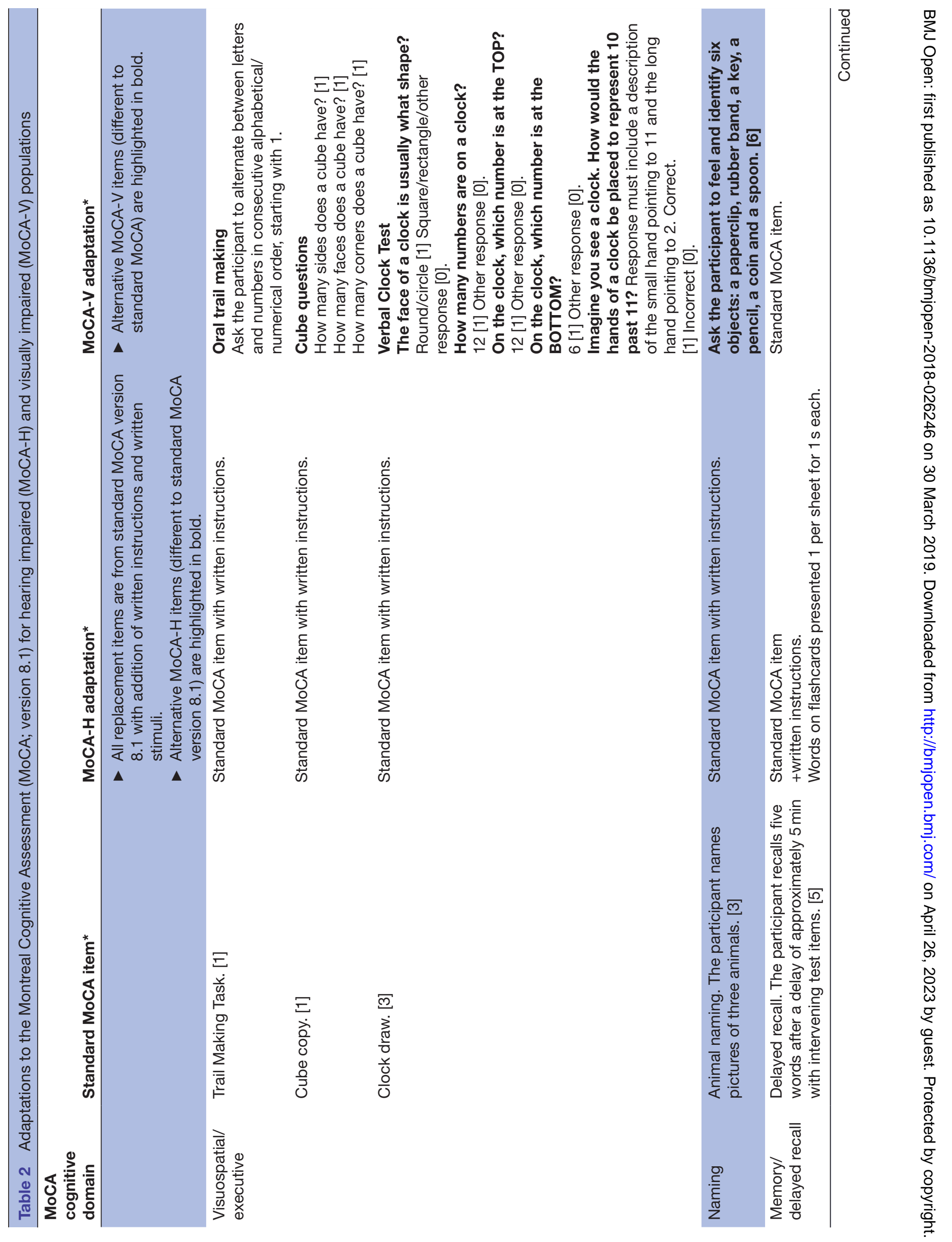




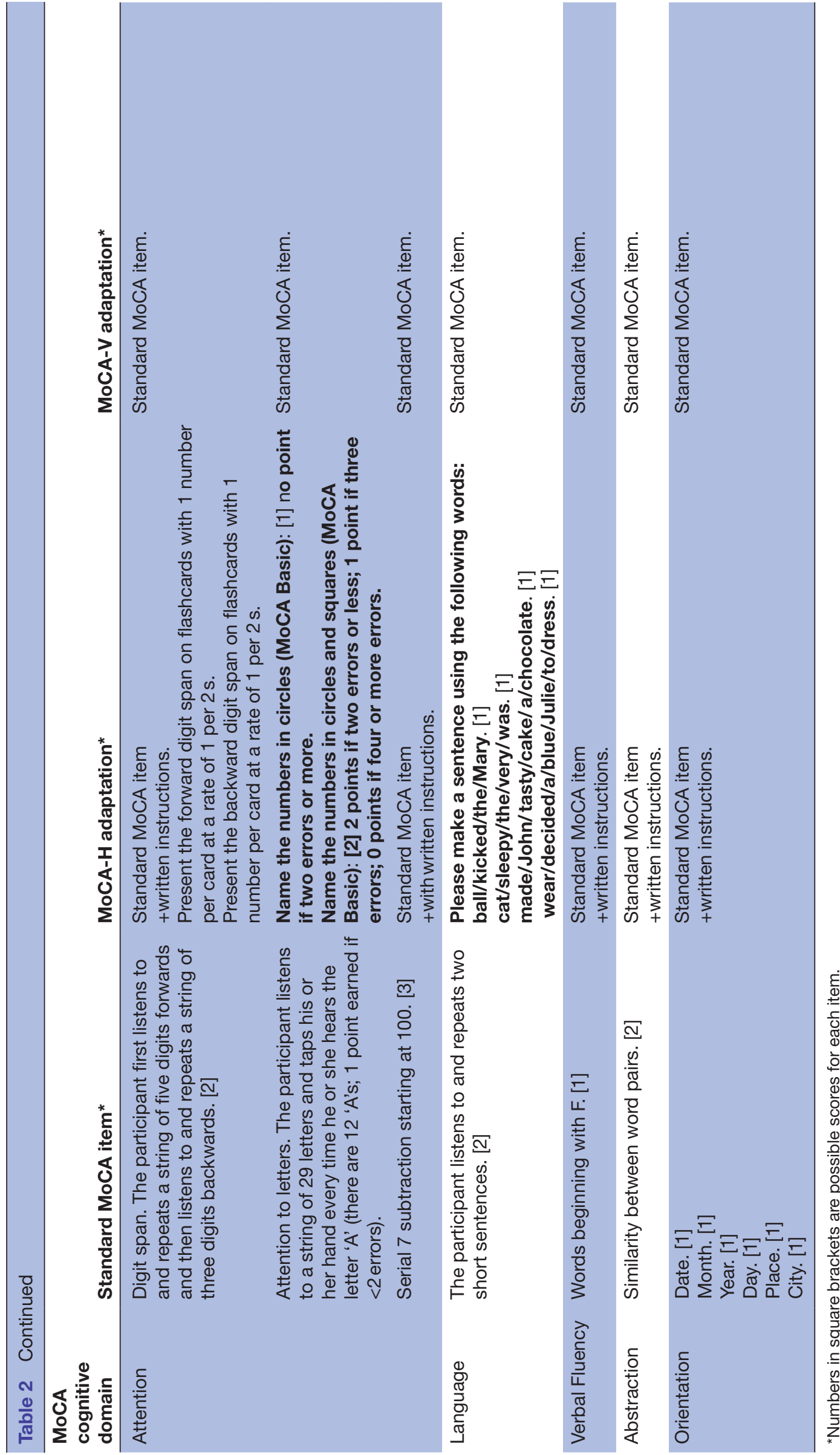


cognitive status (ie, dementia/no dementia). The analysis will be based on the 264 participants with hearing/vision impairment and use a stepwise backwards elimination method for removal of items from the regression model. At the first step, all items that passed the item analysis stage will be entered as a group. At each subsequent step, the item that contributes least to the explanatory power of the model (the item with the largest $\mathrm{p}$ value) will be removed. This will continue until all items remaining in the model have a $p$ value of 0.1 or lower. We use a high $p$ value $(10 \%)$ at this stage for inclusivity, prior to further assessment.

For verification we will then repeat this analysis but using stepwise entry of items in place of stepwise removal. A final selection of items will be decided through comparison of the two models: where there are differences, a final decision will be made taking account of any relevant theoretical and statistical considerations.

\section{Comparison of models}

As a final step, we will compare the resulting models from the substitution-based and exploratory approaches to constructing the MoCA-H and the MoCA-V. We will compute participant scores on each model by totalling across the correctly answered items, as per the procedure for the standard MoCA. The models will then be compared on a range of key performance indices including AUC, internal consistency (Cronbach's alpha), test-retest reliability (intracluster reliability coefficient), sensitivity and specificity, and optimum cut-point for dementia diagnosis. A choice of the final recommended version of the MoCA-H will then be made on the basis of this comparison along with relevant clinical considerations. Assessment at participants' homes may facilitate performance on the 'orientation to place' questions, reduce stress and impact on the total score. Therefore, comparability of scores would be tested with reference to existing MoCA normative data with respect to test site, age and educational level.

The result of the above analytical procedures will be finalised versions of the MoCA-H and MoCA-V instruments, in each of three languages (English, French and Greek) together with recommended threshold values for detecting dementia and measures of internal consistency and test-retest reliability.

\section{Study start and duration}

It is anticipated that data collection will start in June 2018 and run for 18 months.

\section{Ethics and dissemination}

The results of the study will be disseminated through peer-reviewed publication, conference presentations, the study website (https://www.sense-cog.eu/), the SENSE-Cog Twitter account (@sense_cog) and the MoCA test website (https://www.mocatest.org/).

\section{DISCUSSION}

The current paper describes the protocol for the development and validation of versions of the MoCA (version
$8.1)^{11}$ for the identification of dementia within populations of adults with acquired hearing or vision impairment. Six participant groups will complete the MoCA or a version of the MoCA adapted to accommodate either vision or hearing impairment-the MoCA-H and the MoCA-V. Through a process of item and predictive analyses, we will determine the combinations of items with the best balance of discriminative power relative to gold standard diagnostic criteria, clinical validity and utility and reliability, within groups of adults with hearing or vision impairment.

The development of the MoCA-H and the MoCA-V draws on the diagnostic strengths of the previously well-validated MoCA. It is anticipated that through item substitution rather than the deletion of items, the MoCA-H for people with hearing impairment and the MoCA-V for people with vision impairment will have superior validity and reliability compared with previously adapted alternative measures. ${ }^{10}$

The primary limitations of the present study are twofold. First, due to the complexity of design and the large numbers of participants with specific combinations of cognitive and sensory impairments required, it was not feasible to include a group of participants with MCI in addition to normal cognition and dementia groups. The authors are currently seeking additional resources to support addition of an MCI group to the validation sample. Second, the MoCA-H and the MoCA-V that will be developed in the present study rely either on good vision or good hearing. Neither test is suitable for those with dual sensory impairment. Around $1.5 \%$ of adults aged over 20 years has a dual sensory impairment (best-corrected better eye visual acuity $>0.30[6 / 12,20 / 40]$ ) and better ear threshold $>25 \mathrm{~dB}$ HL across $0.5-4 \mathrm{kHz}$ ) ${ }^{32}$ so development of suitable cognitive screening tests for those with dual sensory impairment is important. Given the reliance of MoCA items on either hearing or vision function, the MoCA test paradigm is not suitable for adaptation for those with dual sensory impairment. Alternative cognitive screening tests for those with dual sensory impairment are available or in development, based, for example, on touch $^{33} 34$ or smell. ${ }^{35}$

Outputs for the present study will include adaptations of the MoCA suitable for use in people with hearing and vision impairments. In addition to this, the study will provide validation data on Greek and French versions of the MoCA (version 8.1) in populations without sensory impairment.

\section{Author affiliations}

${ }^{1}$ Manchester Centre for Audiology and Deafness, University of Manchester, Manhcester, UK

${ }^{2}$ Institute of Brain, Behavior and Mental Health, University of Manchester, Manchester, UK

${ }^{3}$ Institute of Population Health, Centre for Biostatistics, University of Manchester, Manchester, UK

${ }^{4}$ Division of Neuroscience and Experimental Psychology, University of Manchester, Manchester, UK

${ }^{5}$ Department of Health Sciences, European University Cyprus, Nicosia, Cyprus 
${ }^{6}$ MoCA Clinic \& Institute, Quebec, Canada

${ }^{7}$ Manchester Academic Health Sciences Centre, Institute of Brain, Behaviour, and Mental Health, Manchester, UK

Contributors IL and PD are responsible for the overall development of an ethically sound protocol. PD, KG, AP, SS, CT, APC, WKY and ZN developed the Montreal Cognitive Assessment - Hearing impaired version (MoCA-H) and Montreal Cognitive Assessment - Vision impaired version (MoCA-V). PD, AP and DR designed the validation study, and DR planned the analyses. All authors contributed to the drafting, critical revision and final approval of the document. Thank you to the patient panels who provided advice on the design and conduct of this research.

Funding This work was supported by the European Union's Horizon 2020 research and innovation programme under grant agreement number 668648 . PD is supported in part by the National Institute for Health Research Manchester Biomedical Research Centre.

Competing interests None declared.

Patient consent for publication Not required.

Ethics approval This study has been reviewed by local ethics committees in the UK, Cyprus, France and Greece. Ethical approvals were granted by the Greater Manchester West Research Ethics Committee (UK) on 13 September 2017, by the Cyprus National Bioethics Committee on 19 January 2017, by the Comité de Protection des Personnes du Sud-Ouest et Outre-Mer IV on 25 May 2018 and by the Local Ethical Committee of Health Sciences and Scientific Committee of the Eginition Hospital of the National and Kapodistrian University of Athens on 15 December 2017.

Provenance and peer review Not commissioned; externally peer reviewed.

Open access This is an open access article distributed in accordance with the Creative Commons Attribution Non Commercial (CC BY-NC 4.0) license, which permits others to distribute, remix, adapt, build upon this work non-commercially, and license their derivative works on different terms, provided the original work is properly cited, appropriate credit is given, any changes made indicated, and the use is non-commercial. See: http://creativecommons.org/licenses/by-nc/4.0/.

\section{REFERENCES}

1. Uhlmann RF, Teri L, Rees TS, et al. Impact of mild to moderate hearing loss on mental status testing. Comparability of standard and written Mini-Mental State Examinations. J Am Geriatr Soc 1989;37:223-8.

2. Reischies FM, Geiselmann B. Age-related cognitive decline and vision impairment affecting the detection of dementia syndrome in old age. Br J Psychiatry 1997;171:449-51.

3. Wittich W, Phillips N, Nasreddine ZS, et al. Sensitivity and specificity of the montreal cognitive assessment modified for individuals who are visually impaired. J Vis Impair Blind 2010;104:360-8.

4. Dupuis K, Pichora-Fuller MK, Chasteen AL, et al. Effects of hearing and vision impairments on the montreal cognitive assessment. Neuropsychol Dev Cogn B Aging Neuropsychol Cogn 2015;22:413-37.

5. Jorgensen LE, Palmer CV, Pratt S, et al. The effect of decreased audibility on MMSE performance: a measure commonly used for diagnosing dementia. J Am Acad Audiol 2016;27:311-23.

6. Wood J, Chaparro A, Anstey K, et al. Simulated visual impairment leads to cognitive slowing in older adults. Optom Vis Sci 2010;87:1037-43.

7. Kempen JH, Krichevsky M, Feldman ST. Effect of visual impairment on neuropsychological test performance. J Clin Exp Neuropsychol 1994;16:223-31.

8. Gold M, Lightfoot LA, Hnath-Chisolm T. Hearing loss in a memory disorders clinic. A specially vulnerable population. Arch Neurol 1996;53:922-8

9. Bowen M, et al. The Prevalence of Visual Impairment in People with Dementia (the PrOVIDe study): a cross sectional study of 60-89 year old people with dementia and qualitative exploration of individual, carer and professional perspectives. Southampton: National Institute of Health Research, 2016.

10. Pye A, Charalambous AP, Leroi I, et al. Screening tools for the identification of dementia for adults with age-related acquired hearing or vision impairment: a scoping review. Int Psychogeriatr 2017;29:1771-84.

11. Nasreddine ZS, Phillips NA, Bédirian V, et al. The Montreal Cognitive Assessment, MoCA: a brief screening tool for mild cognitive impairment. J Am Geriatr Soc 2005;53:695-9.

12. Freitas S, Simões MR, Alves L, et al. Montreal Cognitive Assessment (MoCA): validation study for frontotemporal dementia. J Geriatr Psychiatry Neurol 2012;25:146-54.

13. Hoops S, Nazem S, Siderowf AD, et al. Validity of the MoCA and MMSE in the detection of $\mathrm{MCl}$ and dementia in Parkinson disease. Neurology 2009;73:1738-45.

14. Freitas S, Simões MR, Alves L, et al. Montreal cognitive assessment: validation study for mild cognitive impairment and Alzheimer disease. Alzheimer Dis Assoc Disord 2013;27:37-43.

15. Lin VY, Chung J, Callahan BL, et al. Development of cognitive screening test for the severely hearing impaired: Hearing-impaired MoCA. Laryngoscope 2017;127 Suppl 1:S4-S11.

16. Cha ES, Kim KH, Erlen JA. Translation of scales in cross-cultural research: issues and techniques. J Adv Nurs 2007;58:386-95.

17. World Health Organization. The ICD-10 classification of mental and behavioural disorders: clinical descriptions and diagnostic guidelines. 1992;1.

18. Brodaty $\mathrm{H}$, Pond D, Kemp NM, et al. The GPCOG: a new screening test for dementia designed for general practice. J Am Geriatr Soc 2002;50:530-4.

19. Lobo A, et al. Prevalence of dementia and major subtypes in Europe: a collaborative study of population-based cohorts. Neurology 2000;54:S4.

20. National Institute for Health Research. Join dementia research. 2018. https://www.joindementiaresearch.nihr.ac.uk/.

21. Alzheimer Europe. Decision making and legal capacity in dementia. 2016. https://www.alzheimer-europe.org/Policy-in-Practice2/ Country-comparisons/2016-Decision-making-and-legal-capacity-indementia (cited 1 Jul 2019).

22. National Institute for Health Research. Good clinical practice. 2018. https://www.nihr.ac.uk/our-faculty/clinical-research-staff/learningand-development/national-directory/good-clinical-practice/.

23. Miah J, Dawes P, Leroi I, et al. A protocol to evaluate the impact of involvement of older people with dementia and age-related hearing and/or vision impairment in a multi-site European research study. Res Involv Engagem 2018;4.

24. University of Manchester. Enhancing the Quality of User Involved Care Planning in Mental Health Services (EQUIP). 2012. http:// research.bmh.manchester.ac.uk/equip (cited 2 Apr 2018).

25. Frank T, Durrant JD, Lovrinic JM. Maximum permissible ambient noise levels for audiometric test rooms. Am J Audiol 1993;2:33-7.

26. Folstein MF, Folstein SE, McHugh PR. "Mini-mental state". A practical method for grading the cognitive state of patients for the clinician. J Psychiatr Res 1975;12:189-98.

27. Brodaty $\mathrm{H}$, Connors $\mathrm{MH}$, Loy $\mathrm{C}$, et al. Screening for dementia in primary care: A comparison of the GPCOG and the MMSE. Dement Geriatr Cogn Disord 2016;42(5-6):323-30.

28. Basic D, Khoo A, Conforti D, et al. Rowland universal dementia assessment scale, mini-mental state examination and general practitioner assessment of cognition in a multicultural cohort of community-dwelling older persons with early dementia. Aust Psychol 2009;44:40-53.

29. De Silva ML, McLaughlin MT, Rodrigues EJ, et al. A minimental status examination for the hearing impaired. Age Ageing 2008;37:593-5

30. Kaemmerer T, Riordan P. Oral adaptation of the trail making test: $\mathrm{A}$ practical review. Appl Neuropsychol Adult 2016;23:384-9.

31. Cercy SP. The verbal clock test: Preliminary validation of a brief, vision- and motor-free measure of executive function in a clinical sample. Clin Neuropsychol 2012;26:1312-41.

32. Swenor BK, Ramulu PY, Willis JR, et al. The prevalence of concurrent hearing and vision impairment in the United States. JAMA Intern Med 2013;173:312-3.

33. Kehrberg KL, Kuskowski MA, Mortimer J, et al. Validating the use of an enlarged, easier-to-see allen cognitive level test in geriatrics. Phys Occup Ther Geriatr 1993;10:1-14

34. Bruhn P, Dammeyer J. Assessment of dementia in individuals with dual sensory loss: application of a tactile test battery. Dement Geriatr Cogn Dis Extra 2018;8:12-22.

35. Gros A, Manera V, De March CA, et al. Olfactory disturbances in ageing with and without dementia: towards new diagnostic tools. $J$ Laryngol Otol 2017;131:572-9. 